\title{
A PRECISE, LOW-COST RTK GNSS SYSTEM FOR UAV APPLICATIONS
}

\author{
W. Stempfhuber and M. Buchholz \\ Beuth Hochschule für Technik Berlin, University of Applied Sciences, Department III, Luxemburger Str. 10, \\ D-13353 Berlin, Germany, stempfhuber@beuth-hochschule.de, michael.buchholz@beuth-hochschule.de
}

KEY WORDS: RTK GNSS, Low-cost, Kinematic, UAVs, Machine Control, Single-Frequency-Receiver

\begin{abstract}
:
High accuracy with real-time positioning of moving objects has been considered a standard task of engineering geodesy for 10 to 15 years. An absolute positioning accuracy of 1-3 cm is generally possible worldwide and is further used in many areas of machine guidance (machine control and guidance), and farming (precision farming) as well as for various special applications (e.g. railway trolley, mining, etc.). The cost of the measuring instruments required for the use of geodetic L1/L2 receivers with a local reference station amounts to approximately USD 30,000 to 50,000. Therefore, dual frequency RTK GNSS receivers are not used in the mass market.
\end{abstract}

Affordable GPS/GNSS modules have already reached the mass market in various areas such as mobile phones, car navigation, the leisure industry, etc. Kinematic real-time positioning applications with centimetre or decimetre levels could also evolve into a mass product. In order for this to happen, the costs for such systems must lie between USD 1,000 to 2,000. What exactly low-cost means is determined by the precise specifications of the given individual application.

Several university studies in geodesy focus on the approach of high-accuracy positioning by means of single frequency receivers for static applications [e.g. GLABSCH et. al. 2009, SCHWIEGER and GLÄSER 2005, ALKAN 2010, REALINI et. al. 2010, KORTH and HOFMANN 2011]. Although intelligent approaches have been developed that compute a trajectory in the post-processing mode [REALINI et. al., 2010], at present, there are only a very few GNSS Low-Cost Systems that enable real-time processing.

This approach to precise position determination by means of the computation of static raw data with single frequency receivers is currently being explored in a research project at the Beuth Hochschule für Technik Berlin - and is being further developed for kinematic applications. The project is embedded in the European Social Fund. It is a follow-up project in the area of static positioning with single GNSS frequency receivers [KORTH and HOFMANN, 2011].

\section{MOTIVATION}

The exact positioning of moving objects within an accuracy range of a few centimetres has been possible through geodetic measuring sensors for approximately 15-20 years. With this, one can distinguish between the systems for 3D-capture in terrestrial (Total Stations or IMUs) and global processes (GNSS). Nowadays, many applications for exact positioning utilise geodetic RTK GNSS receivers. GPS single frequency receivers with or without code corrections and with lower accuracy standards are used for this. Today and as a rule, kinematic applications at the centimetre level use geodetic 2 frequency receivers for the determination of a 3D-trajectory. Many GNSS applications are not realisable due to the high cost and the heavy weight of the measuring equipment. Therefore today, the usage of RTK GNSS primarily limits itself to applications in the areas of the machine control and guidance as well as Precision Farming.

At the Beuth Hochschule für Technik Berlin - University of Applied Sciences and within the scope of a research project, the capabilities of single frequency receivers are verified and then adapted and developed for special applications. This modular GNSS System (see Figure 2) is based on the solution of the L1-carried phase ambiguity. It is able to calculate centimetreexact, 3D-positions on a real-time basis and be output to in interfaces. With this, different real-time processes can be realised. An example is the control and guidance of small Mindstorms NXT-model vehicle. Subsequent system structures are necessary for this purpose. 


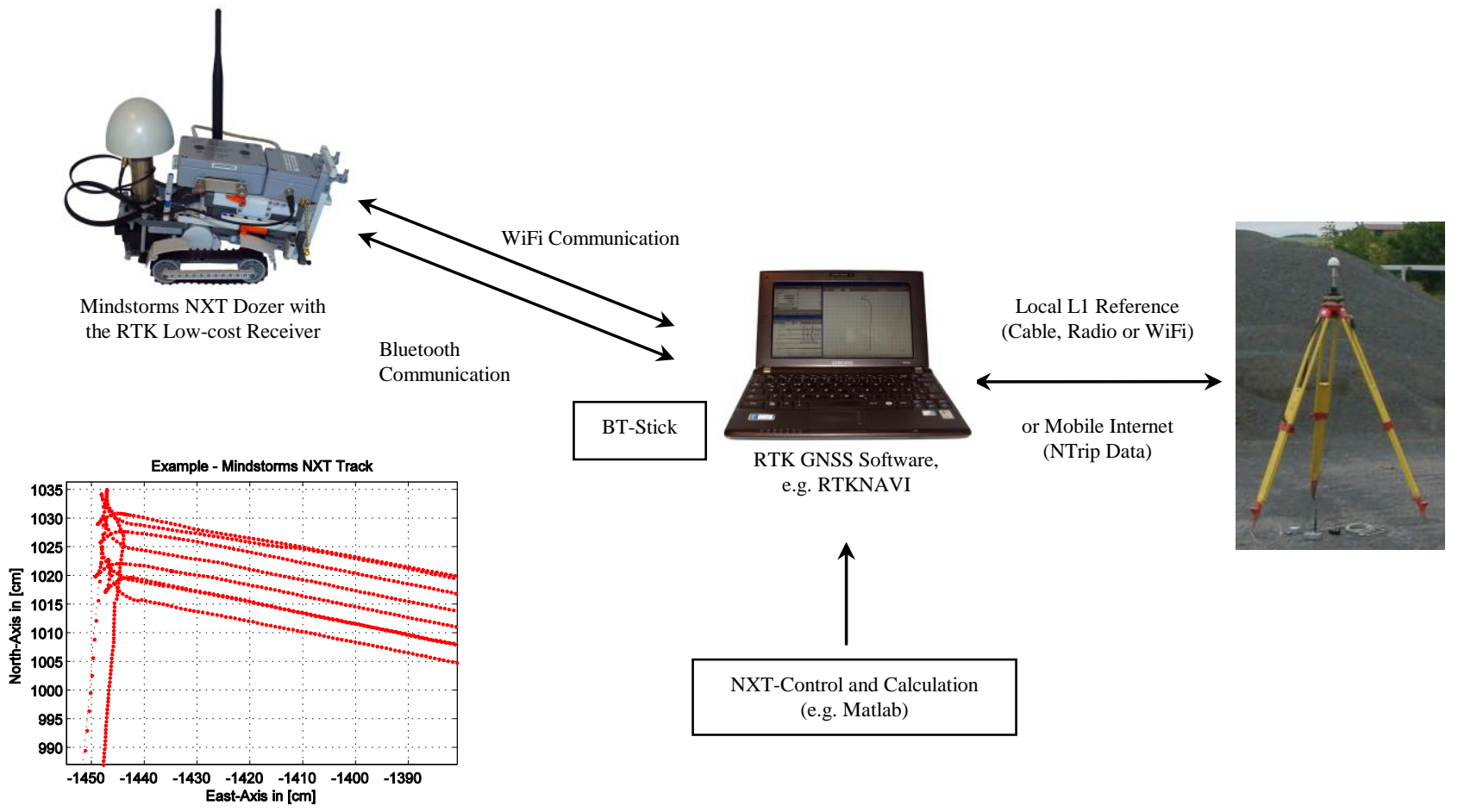

Figure 1. RTK Mindstorms NXT-Control via the RTK GNSS Low-cost System

These models are able to conduct target tracking on a real-time basis. Through the integration of other Mindstorms sensors, such movement simulators can simulate actual applications very realistically. This is currently being conducted in various, small projects at the Beuth Hochschule für Technik Berlin University of Applied Sciences.

\section{SYSTEM COMPONENTS OF THE LOW-COST RTK GNSS}

As a general rule, commercial GNSS products are available to the user as a complete system. The approach of the research project for the real-time solution and output of the L1-carried phase ambiguity is based on a modular hard and software approach. Aside from the various implemented GNSS boards (z.B. ublox, Sky Traq, NovaTel, Hemisphere, Superstar, etc.) and the different GNSS aerials, first and foremost, the RTK software is a central component. More than 10 years ago, a Matlab Freeware with the project name GPSLab [Zebhauser,
2000] was already available for static GPS measurements. Since 2006, the Japanese university project, RTKLIB, is freely assessable for real-time applications [Takasu, 2008]. RTKLIB is an Open Source software package for GNSS positioning. With this programme, one can process and store raw GPS data on a real-time basis as well as in post-processing. The software package consists of several partially independent program components. These are following modules:

- RTKNavi GNSS Realtime Calculation and NMEA Output,

- RTKPost Post-processing Software,

- RTKPlot Visualisation,

- RTKConv Convert to RINEX Data and

- $\operatorname{pos} 2 \mathrm{kml}$ Create a Keyhole Markup Language (KML)-Dataset

The solution of the L1-carried phase ambiguity uses the approach of [Teunissen 1995] and [Chang 2005]. Building on this component, overall systems were subsequently developed with the Research Assistance V and VI. 

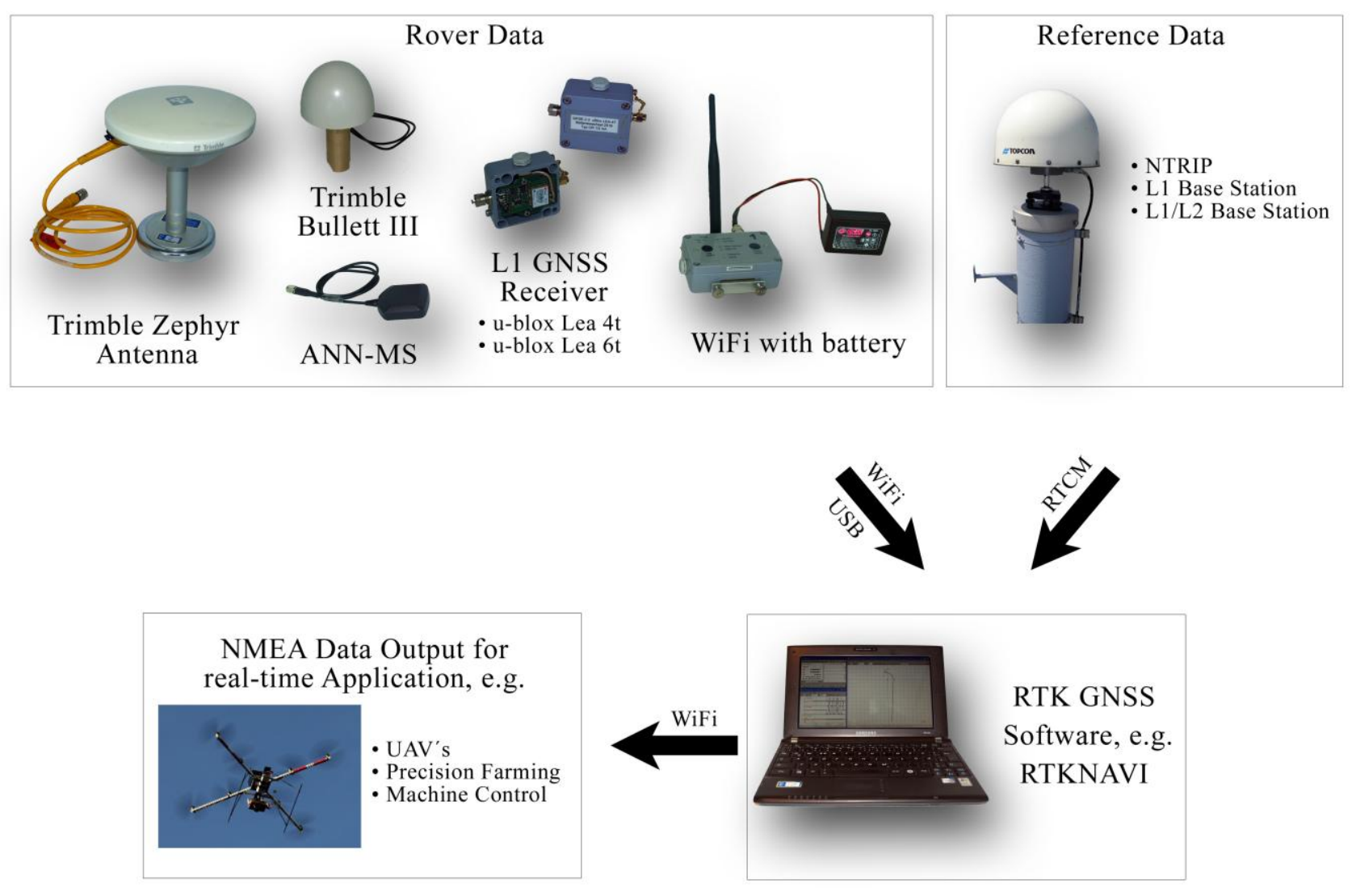

Figure 2. RTK GNSS Low-cost System Components

As a result, all hard and software modules could be exchanged. The first battery of testing has shown that under good conditions (e.g. with the ublox LEA 4T or 6T), this system ran a nearly complete fix ambiguity solution after an initialization time of a few minutes. As a result, the reference information can be applied by different processes e.g. RTCM via NTRIP or a local RTCM data stream. Certainly here, the use of a second low-cost receiver (similar to the rover) is the most economical solution. The widely-known GNSS disturbance variables can also be observed more precisely with this approach. As a rule, if the system loses the fix ambiguity solution, the satellite signals are disturbed through Multipath. Our own Choke-ring antenna was designed to minimise this effect [Korth 2010 or Stempfhuber 2011].
For kinematic application, this system must be tested on moving objects and adapted. The first series of tests was conducted with the company Moba (www.moba.de). The test construction with two ublox LEA 6T boards and two Trimble Bullet III L1-antennas for the reference and rover receivers is based on the designs in Figures 1 and 2. With a movement velocity of approximately $1 \mathrm{~m} / \mathrm{s}$, this test set-up rendered a complete fix ambiguity solution on a real-time basis after an initialization time of $\sim 180 \mathrm{sec}$.

In addition, further different system components (with variable configuration and hardware components) were verified under changed conditions. 

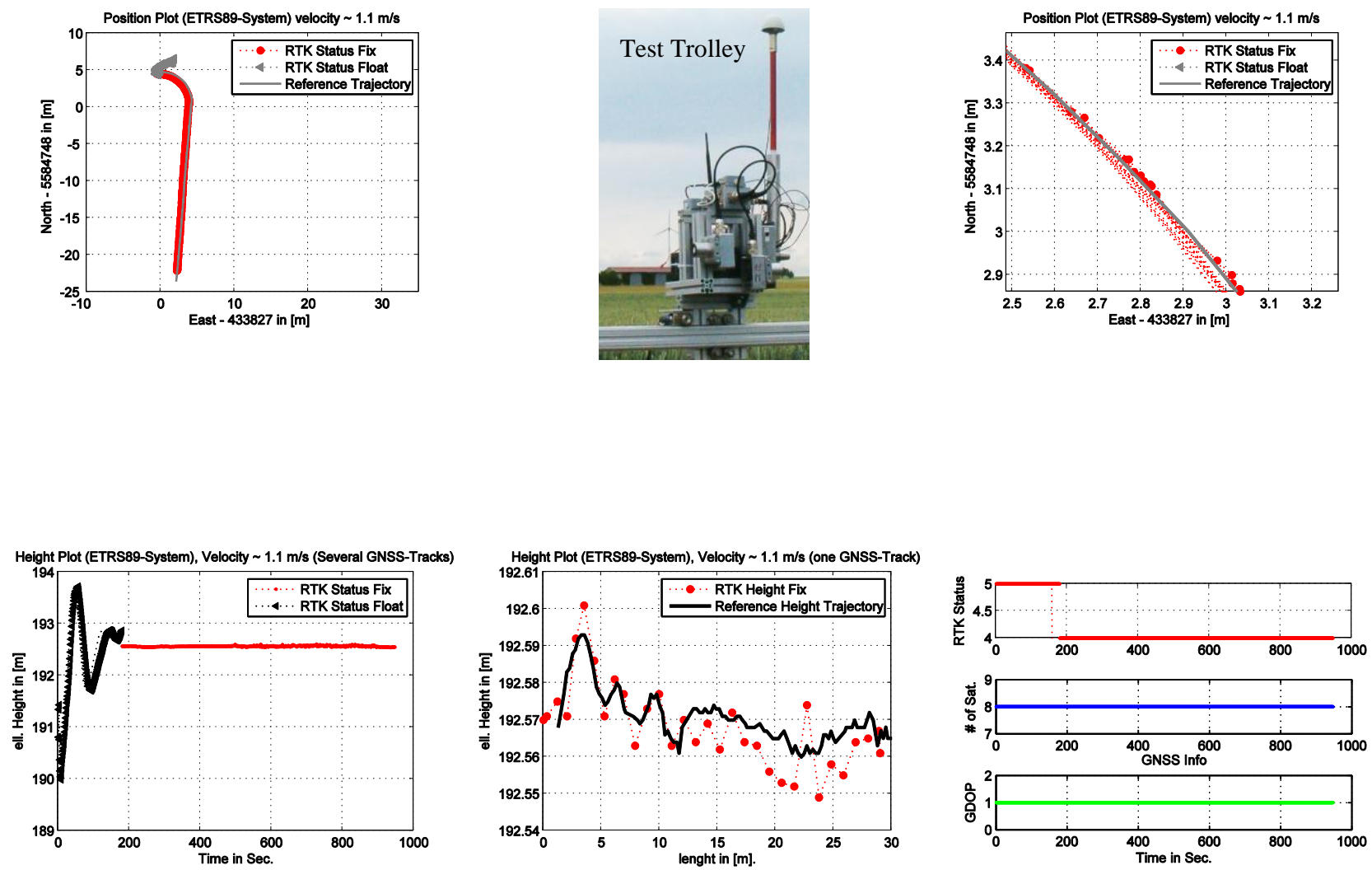

Figure 3. Results of the first Test Measurement at Moba

\section{PRECISE 3D-POSITIONING OF UAVS}

At the moment, nearly all GPS-supported UAV applications are being used as a stand-alone or as a differential corrected code solutions. Therefore, an absolute positioning of a moving model helicopter was only possible in metres under good conditions at the sub-metre level. The exact analysis and referencing of the aerial image data still occurs through the use of exact control points. This limits many UAV applications. Through the low weight and the low cost of the low-cost RTK GNSS-System, existing UAV GNSS receivers can be easily substituted with these GNSS prototypes. The real-time data output occurs via the standardised NMEA output protocol. Subsequent test construction could be very simply realised for the initial test.
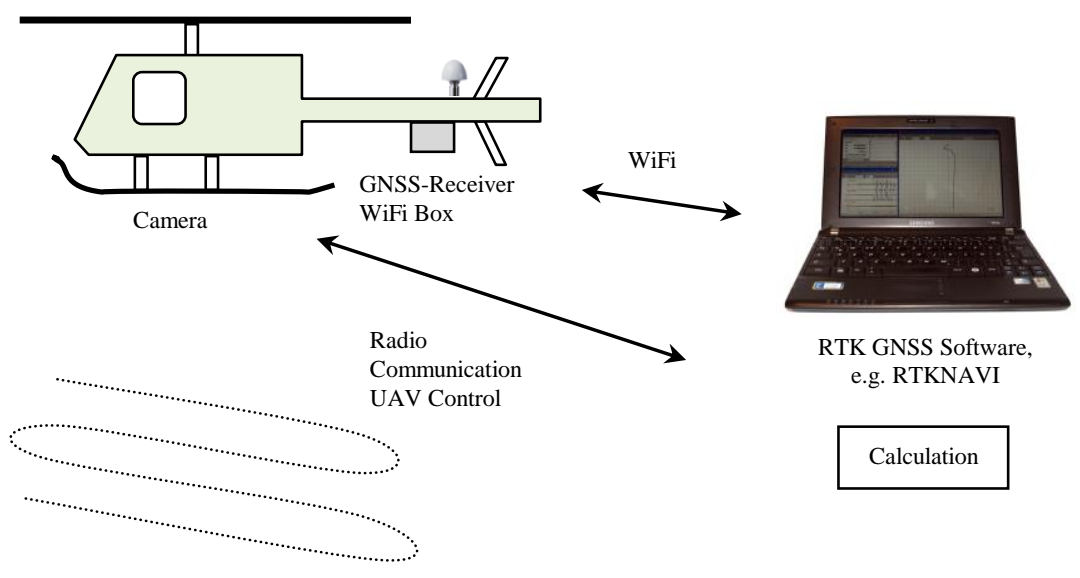

Local L1 Reference (Cable, Radio or WiFi)

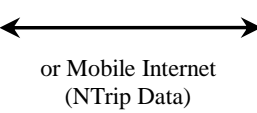

(NTrip Data)

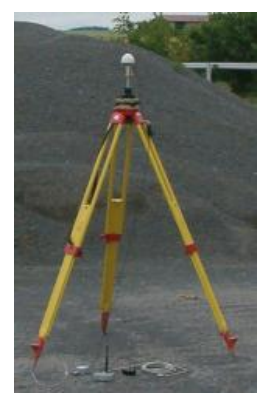

3D-Trajectory

Figure 4. RTK GNSS Low-cost System based UAV-Control 
Initial tests are planned concerning the 3D-directional stability with different UAV systems. The 3D-trajectory of UAVs with code-corrected GPS had already been verified with targettracking total station [Eisenbeiss and Stempfhuber, 2009]. With an economical and very light L1 receiver (weight: ublox receiver incl. ports and a waterproof housing $193 \mathrm{~g}$, the Bullet III antenna is $179 \mathrm{~g}$ plus the WiFi module incl. the data logger with around $350 \mathrm{~g}$ ), this described approach could position the absolute 3D-position in the area of a few centimetres on a realtime basis.

\section{OUTLOOK}

Through this described system and the Open-Source Code RTKNavi Calculation Software for the real-time solution of the carried phase ambiguity, the possibility exists to control any land-based or airborne objects on a real-time basis. In addition, the analysis and the re-initialization can be optimised for the appropriate motion sequences. Moreover, only the a priori information from the flight planning and the airspeed are necessary. Therefore, an efficient and stable positioning is possible in addition to the RTKNavi parameter [Takasu, 2008]. From the modular system structure (different GNSS boards, GNSS aerials, correction services, etc.), the influence of disturbances can be minimised for each, individual application and therefore, the overall system can be optimised. Presently, this is being carried out on various applications in the described research project. The availability of a stable fix ambiguity solution can additionally be achieved through the introduction of GLONASS on these small and economical OEM-Boards.

\section{References}

Alkan, R. M.:, Development of a Low-cost Positioning System Using OEM GPS Receivers and Usability in Surveying, Applications , FIG Congress 2010 Facing the Challenges Building the Capacity, Sydney, 2010.

Chang, X.-W., Yang, X. and Zhou, T.: MLAMBDA - A modified LAMBDA method for integer least-squares estimation, J. Geodesy, vol.79, 2005.

Eisenbeiss, H. Stempfhuber, W. and Kolb, M.: Genauigkeitsanalyse der 3D-Trajektorie von Mini-UAVs, Tagungsband der Deutschen Gesellschaft für Photogrammetrie und Fernerkundung (DGPF) 18/2009.

Glabsch, J., Heunecke, O. and Schuhbäck, S.:

Hangüberwachungen mittels Low Cost GNSS im alpinen Raum - Ansätze und Erfahrungen, ETH Zürich, AHORH 2009.

Korth, W. and Hofmann, U.: Softwareentwicklung für Positionsbestimmung mit Satelliten, Abschlussbericht Forschungsassistenz VI, 2011.

Realini, E., Yoshida, D., Reguzzoni, M. and Raghavan, V.: Testing goGPS Low-Cost RTK Positioning With A Wen-Based Track Log Management System, WebMGS 2010.

Schwieger, V. and Gläser, A.: Possibilities of Low Cost GPS Technology for Precise Geodetic Applications. Proceedings on FIG Working Week 2005, Kairo, 2005.

Stempfhuber, W. and Buchholz, M.: High-End and Low-Cost RTK GNSS in Machine Control and Precision Farming
Applications, FIG Working Week 2011 Bridging the Gap between Cultures, Marrakech, Morocco, 18-22 May 2011.

Takasu, T., and Yasuda A.: Evaluation of RTK-GPS Performance with Low-cost Single-frequency GPS Receivers, International Symposium on GPS/GNSS 2008, November 1114, Tokyo International Exchange Center, Japan 2008.

RTKLIB: An Open Source Program Package for RTK-GPS, http://gpspp.sakura.ne.jp/rtklib/rtklib.htm

Teunissen, P. J. G.: The least-square ambiguity decorrelation adjustment: a method for fast GPS ambiguity estimation, J. Geodesy, vol.70, 1995.

Zebhauser, B.: Zur Entwicklung eines GPS-Programms für Lehre und Tests unter besonderer Berücksichtigung der Ambibuity Function Methode, DKG-Reihe C, Heft Nr. 523, 2000 . 